\title{
Adaptive Path-Finding and Transport Network Formation by the Amoeba-Like Organism Physarum
}

\author{
Itsuki Kunita $^{1}$, Kazunori Yoshihara ${ }^{1}$, Atsushi Tero $^{2}$, Kentaro Ito $^{3}$, \\ Chiu Fan Lee ${ }^{4}$, Mark D. Fricker ${ }^{5}$, and Toshiyuki Nakagaki ${ }^{1,6}$ \\ 1 Department of Complex and Intelligent Systems, \\ Faculty of Systems Information Science, Future University of Hakodate, \\ 116-2 Kamedanakano-cho, Hakodate, Hokkaido, Japan 041-8655 \\ 2 Institute of Mathematics for Industry, Kyushu University, \\ 744 Motooka, Nishi-ku, Fukuoka 819-0395, Japan \\ 3 Department of Mathematical and Life Sciences, \\ Faculty of Science, Hiroshima University \\ 4 Department of Bioengineering, \\ Imperial College London, London, SW7 2AZ, UK \\ 5 Department of Plant Science, University of Oxford, \\ Parks Road, Oxford, OX1 3RB, UK \\ 6 JST, CREST, 5, Sanbancho, Chiyoda-ku, Tokyo, Japan 102-0075 \\ nakagaki@fun.ac.jp
}

\begin{abstract}
The giant amoeba-like plasmodia of Physarum is able to solve the shortest path through a maze and construct near optimal functional networks between multiple, spatially distributed food-sources. These phenomena are interesting as they provide clues to potential biological computational algorithms that operate in a de-centralized, singlecelled system. We report here some factors that can affect path-finding through networks. These findings help us to understand more generally how the organism tries to establish an optimal set of paths in more complex environments and how this behaviour can be captured in relatively simple algorithms.
\end{abstract}

Keywords: Physarum, combinatorial optimization, subcellular computing, primitive intelligence.

\section{Introduction}

The plasmodium of the true slime mold Physarum polycephalum grows as a giant, acellular amoeba, which transports and circulates nutrients and signals through network structure of pulsating tubular veins. The network can reorganize within hours in response to external stimulation and environmental changes. The first evidence that Physarum could connect multiple separate food-sources (FSs) through a tubular network was reported in 1996[1]. The author described that the shape of network seemed to be optimal or quasi-optimal in some physiological sense, and inferred that this might reflect the operation of a biological 
algorithm to compute the optimal network solution. Thus studying network development in organisms such as Physarum might provide the key to understanding how information processing can be performed in cellular system, heralding the advent of bio-inspired computation.

In 2000, Physarum was shown to be able to solve the shortest path through a maze [2 4]. When the organism was allowed to completely fill a maze with two FSs placed at the exits, it rapidly migrated towards the FSs and colonized them, leaving a single thick tube connecting the FSs by the shortest path. This adaptive re-distribution of it's cell body enabled the organism to maximize contact with the FSs, to absorb nutrients as fast as possible, whilst retaining connectivity and communication throughout it's length. A simple mathematical model was proposed to capture this behaviour, based on physiological mechanism linking adaptive development of each tubular element to the mass flow passing through the tube itself [5, 6]. At this stage, the experiments showed that the plasmodium was able to distinguish between paths of relatively similar length, but the level of discrimination was not rigorously determined.

In 2004, the computational ability to find a wide range of near-optimal solutions was reported for networks connecting many FSs distributed in a plane space [7, 8]. The experimental systems represent multi-objective optimization problems, and the solutions found by Physarum approximated a Pareto frontier in the space spanned by multiple evaluation-functions.

Given that the computational ability of Physarum to solve such geometric problems could be expressed in relatively simple mathematical model, a new method for designing a social transport network inspired by Physarum-type algorithms was proposed at the international conference on Dynamical Systems organized by Society of Industrial and Applied Mathematics (SIAM) in May 2005. As an concrete example, the ability of Physarum to design a railway network around Tokyo was examined, using the distribution of FSs to reflect the geographical pattern of major cities in Tokyo region. Physarum was able to make a multi-functional network that connected all the locations with a balance between the total length of the network, the shortest distance between "cities" and the level of fault tolerance to disconnection. Such Physarum-type algorithms were also applied to other graph problems, including navigation through a road networks in response to spatio-temporal variations of congestion, and the problem of finding the Steiner Minimum Tree across a network [9 13]. Physarum computing thus represents a new method of bio-inspired problem-solving and has attracted considerable attention from engineers, scientists and the wider community.

Despite this promising start, only a limited range of experimental maze and network configurations have been tested so far, and our current knowledge of the complex behaviour of this fascinating organisms is still limited. This report examines adaptive responses to more complex, but well specified geometrical mazes and network challenges. In particular, we start to explore configurations where the plasmodium was challenged with multiple parallel routes that differed in length and tortuosity (Fig. 1). 


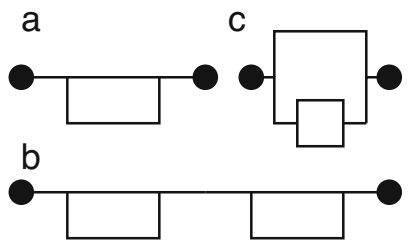

Fig. 1. Schematic diagram showing the topological shapes of mazes used. (a, b) represent the layout used previously and focus on binary decisions based solely on the shortest path length. This paper also explores a new topological configuration with multiple parallel paths that vary in length and tortuosity (c).

\section{Material and Methods}

\subsection{Preparation of Organism and Maze}

The organism we used for experiments was the plasmodium of the true slime mold Physarum polycephalum. Mazes were made as described previously [3]. Previous mazes followed the layout shown in Fig 1a and 1b. Here we tested different topological layouts with multiple parallel paths (see Fig 2a,b). This gave control of the difference in overall length and tortuosity between the different paths. Two configurations were tested: in both shapes, two food-sources were connected through three paths indicated by numbers $a_{1}, a_{2}$ and $a_{3}$. While the path $a_{3}$ was single all the way, paths $a_{1}$ and $a_{2}$ ran parallel to each other over part or all of the route. By varying the location of the FS, indicated by black dots, the relative length of path $a_{3}$ was varied with respect to $a_{2}$ and $a_{3}$. The length difference, termed $\alpha$, was given by (length of $a_{1}$ )/(length of $a_{3}$ ), where the lengths of $a_{1}$ and $a_{2}$ were always the same. It should also be noted that the actual conductivity through tubes of different lengths was also strongly dependent on their thickness, as conductivity follows the Hagen-Poisueille flow approximation. In Fig. 2a, the number of turning points varied between the different paths, whilst in fig. $2 \mathrm{~b}$, the number of turning points was the same. In addition, two dead-end paths were included to ensure that the same initial volume of plasmodium was present in each arm of the maze at the start.

\subsection{Comparison of Network Structure between Real Transport Networks in the Hokkaido Region, Japan, and Physarum Networks Connecting FSs}

Twenty-four FSs were set out to represent the geographical locations of the major towns in the Hokkaido region of northern part of Japan (see Fig. 5), following the method described previously [11].

Three recipes were used to prepare the FSs: (1) each FS was formed from a small portion of kneaded dough that included ground oat flake powder and water only, with all FSs being equal in size (see Fig. 5a); (2) FSs were made using the 


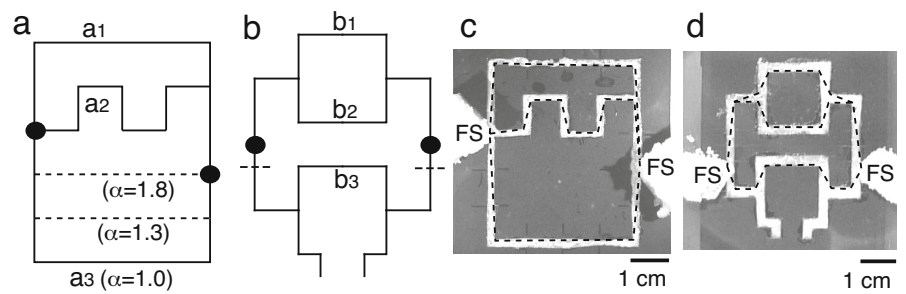

Fig. 2. Two different realizations of the topological layout shown in Fig 1c are shown in (a) and (b) with representative images of the experimental systems showing the typical movement of the Physarum plasmodium shown in (c) and (d), respectively. In (a) the maze has two parallel paths $\left(\mathrm{a}_{1}, \mathrm{a}_{2}\right)$, that are always the same length, and a third, single path $\left(a_{3}\right)$. When two FSs were placed at the locations indicated by black dots in the figure, the length of these three paths is the same (provided the width of the paths is ignored). By varying the position of the FSs, indicated by the dashed lines, the relative length of path $a_{3}$ with respect to $a_{1}$ and $a_{2}$ could be varied. The length difference was characterized as $\alpha=$ (length of $\left.a_{1}\right) /\left(\right.$ length of $\left.a_{3}\right)$. The path $a_{2}$ has 7 turning points, whilst the other two paths have only two. In (b) the maze has the same topology as (a), but the number of turning points is the same in all paths. In addition, to make the initial distribution of the organism the same in all paths, two dead-end paths were included in the lower arm of the maze. (c) shows the distribution of the organism completely filling the maze just after presentation of food-sources (FS) in configuration (a). (d) shows the result of an experiment with configuration (b) after several hours when the organism has accumulated at the FSs and some of the connection paths disappeared. The shortest path remained through the lower arm of the maze, but sometimes additional routes still remained. Scale bar in (c) and (d) indicates $1 \mathrm{~cm}$.

same as recipe (1), but the size of each dough ball was proportional to the size of the population in each town (see Fig. 6e); (3) Each FS was formed from a cubic column of agar gel containing oat flakes. The surface area of the column was proportional to the population of town, since the amount of protoplasm accumulating on the FS is proportional to the surface area, if the concentration of ground oat flakes is equal (see Figs. 6a) [12].

Two additional configurations were included to represent the topographic constraints that would normally apply when considering construction of a rail line in the real world. First, a physical obstacle of columnar blocks of plain agar were introduced to correspond to the four major mountain ranges in Hokkaido (see Fig. 7a). Second, the experimental arena was illuminated with a structured mask, as Physarum is known to avoid high intensity light [14]. A light intensity of $45000 \mathrm{~lx}$ was used to match the position of the four major mountain ranges in the actual Hokkaido landscape (see Fig. 7c). 


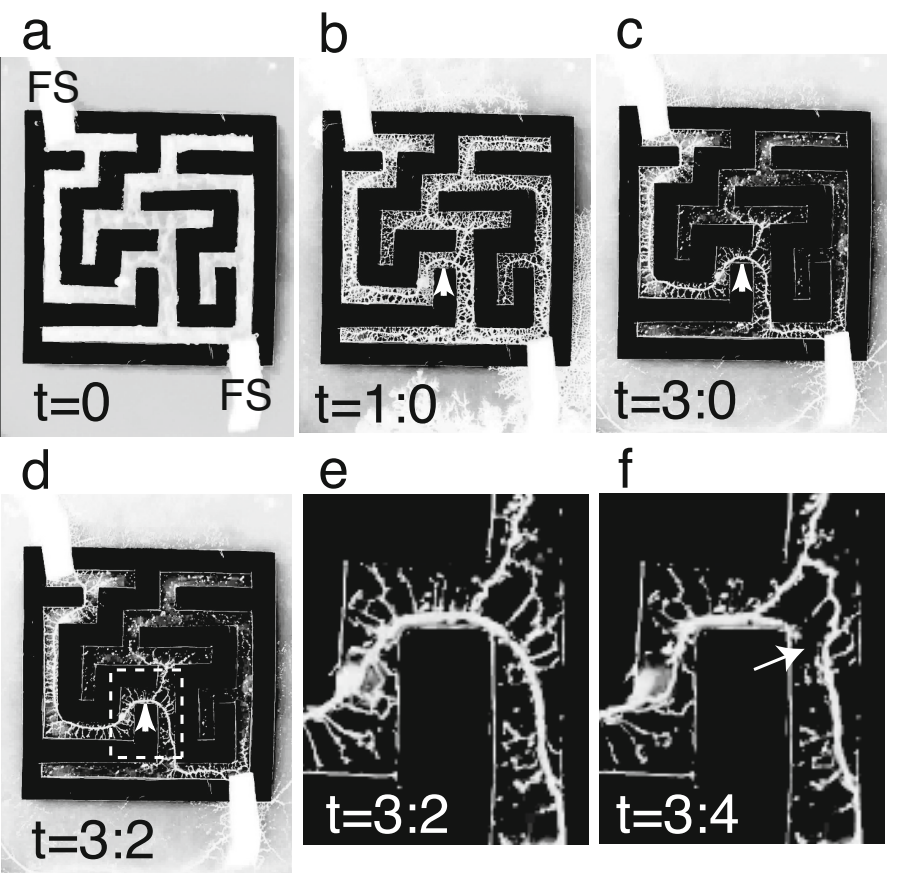

Fig. 3. Solving the maze of topological type a in Fig 1. (a) Top view of the experimental results just after the presentation of the two FSs; (b) at an intermediate state of tube development along the main corridor; and (c, d) at the final state with a single connection through the shortest path. The connection path was stable over a period of time, once the final configuration was reached. In this maze design, there were two possible connection paths between FSs that branched at the point indicated by a white arrow. Interestingly, although the thickest tube was aligned with the shortest path, it did shift a little and then snapped later, as indicated by the white arrow $(e, f)$.

\section{Results}

\subsection{Solving a Maze with the Topological Type in Fig. 1a.}

Figure 3a shows results in a maze with the same topology as used previously, but set out differently. Just after the presentation of FSs (Fig 3a), the Physarum plasmodium began to gather at the FSs and formed some thick veins along the main corridors in the maze (Fig 3b). Eventually, a single thick vein remained that traced the shortest path between the FSs, whilst the other thick veins disappeared (Fig 3c). This results confirmed that the creature can find the shortest path in the maze, given a choice of two alternative routes, irrespective of the actual physical layout of the maze.

To date, most observations suggest that once a tube path is established, the path does not alter its position. However, in this experiment the tube path was able to shift slightly, as indicated by the white arrow in the time course from Fig $3 \mathrm{~b}$ to $3 \mathrm{~d}$. 

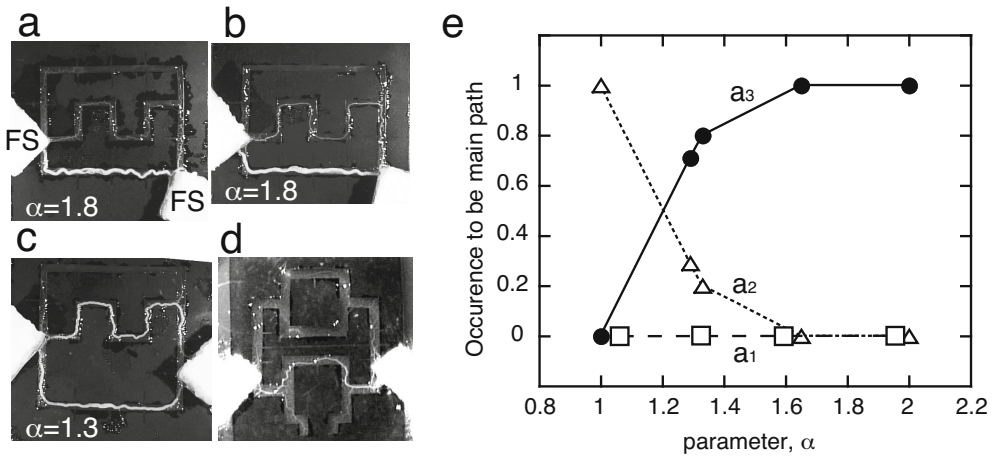

Fig. 4. Statistical occurrence for each path to be main connection between FSs. (a-d) Pictures of the network shape in the maze of Fig. 2a type (a-c) and Fig. 2d type (d). (a) When $\alpha=1.8$, the shortest path $a_{3}$ remained between the two FSs. (b) In some cases in $\alpha=1.8$, the longer path $a_{2}$ was left to connect the FSs but the thickness of $a_{2}$ was thinner than that of the main path $a_{3}$. We classified the connection through $a_{3}$ as main and $a_{2}$ as secondary. The path $a_{2}$ was preferred to the path $a_{1}$ although the length of the two was the same. (c) In some cases in $\alpha=1.3$, the connections through two paths of $a_{2}$ and $a_{3}$ were similar, so both paths were classified as main, although the length of $a_{2}$ was longer. This showed the typical preference of $a_{2}$. (e) Statistical preference to be main path as a function of the parameter $\alpha=\frac{\alpha_{2}}{\alpha_{3}}=\frac{\alpha_{1}}{\alpha_{3}}$.

The thick tube snapped later, as shown in the white arrow in Fig 3f, and the alternative adjacent thin tube instantly became thicker to compensate for the increased the flow. The observation of snapping strongly suggests that tension forces can act along the tube and may cause re-positioning under some circumstances.

\subsection{Solving a Maze with Topological Type in Fig. 1c}

With the introduction of additional parallel pathways, including equidistant routes, the potential range of solutions increases. Figure 4 illustrates results of maze-solving for parallel mazes of the type shown in Fig. 1c and Fig. 2a. The path $a_{3}$ was always selected when the length was sufficiently shorter $(\alpha>1.3)$ than that of the others, where $\alpha=\frac{\alpha_{2}}{\alpha_{3}}=\frac{\alpha_{1}}{\alpha_{3}}$. However, path $a_{2}$ was always selected when all three paths had the same nominal length. Interestingly, path $a_{2}$ was also preferred around $20 \%$ of the time when $\alpha=1.3$, even though it was longer than $\alpha_{3}$, and identical to $\alpha_{2}$ (Fig. 4c). Figure $4 \mathrm{e}$ shows the probability for each route to be selected as the main path as $\alpha$ varies. Moreover, a thin connection through $a_{2}$ was sometimes observed even when $\alpha=1.8$, even though the length was much longer (Fig. 4b). Such thin connections were regarded as secondary rather than main or primary. Another interesting observation was that $a_{1}$ was not preferred at all although the length of $a_{1}$ and $a_{2}$ was the same. The results described above implied that additional factors than length alone are involved in path selection. 

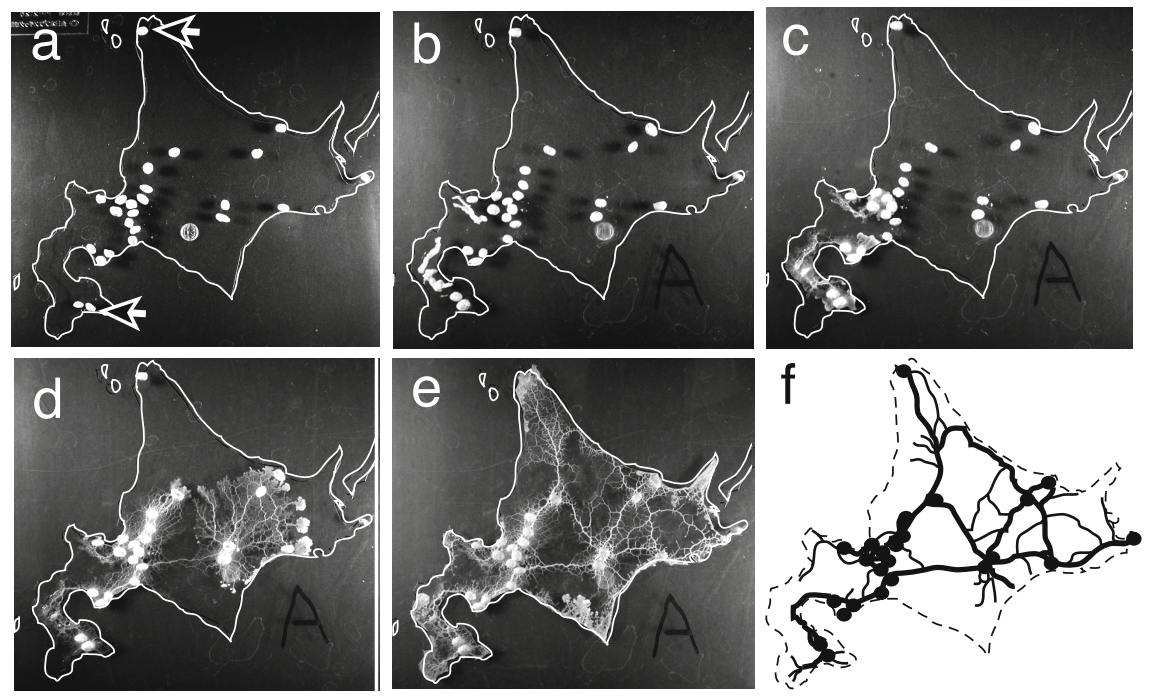

Fig. 5. Time series of the tube-network made by Physarum when challenged with 24 equal sized FSs set out in the geographical pattern of the 24 major towns in Hokkaido. (a) Each FS was formed from a dough of oat flakes. The white arrows indicate the two most remote FSs and the distance between these two FSs was $16 \mathrm{~cm}$. Hokkaido is an island surrounded by the sea, so an acetate sheet was cut out to follow the coastline and constrain the organism to fill the land area. (b) A few pieces of plasmodium were initially inoculated in the low left part of Hokkaido. (c, d) Intermediate state of growing network and (e) the final shape of network. (f) Shows the graph representation of network shape obtained from (e). Thick and thin solid lines indicate thick and thin tubes, respectively. The thick-lines represent the main skeleton of the network.

\subsection{Transport Network in Hokkaido}

Figure 5 shows that Physarum connected all FSs into a network throughout the simulated island of Hokkaido. Initially, a few portions of Physarum plasmodium were put in the lower left part of the arena (Fig. 5b). The organism started to extend out (Fig. 5c) and fused to become a single organism (d). Finally, the Physarum reached all the FSs and connected them with a main skeleton of thick tubes, with a few thinner tubes persisting for a considerable time (e). The network architecture was extracted, after thick tubes and thinner tubes were identified. Figure 5f shows the extracted network, in which thick and thin solid lines indicate the thick and thin tubes, respectively.

Figure 6 shows the tube network connecting FSs with different sizes that scaled in proportion to the population size of each town. A representative typical outcome is shown in Fig. 6c using cubic column of agar gel as FSs and the corresponding network in fig. 6d. In Fig. 6e, the difference in population size was expressed by the size of dough, with the corresponding network shown in Fig. 6f. 

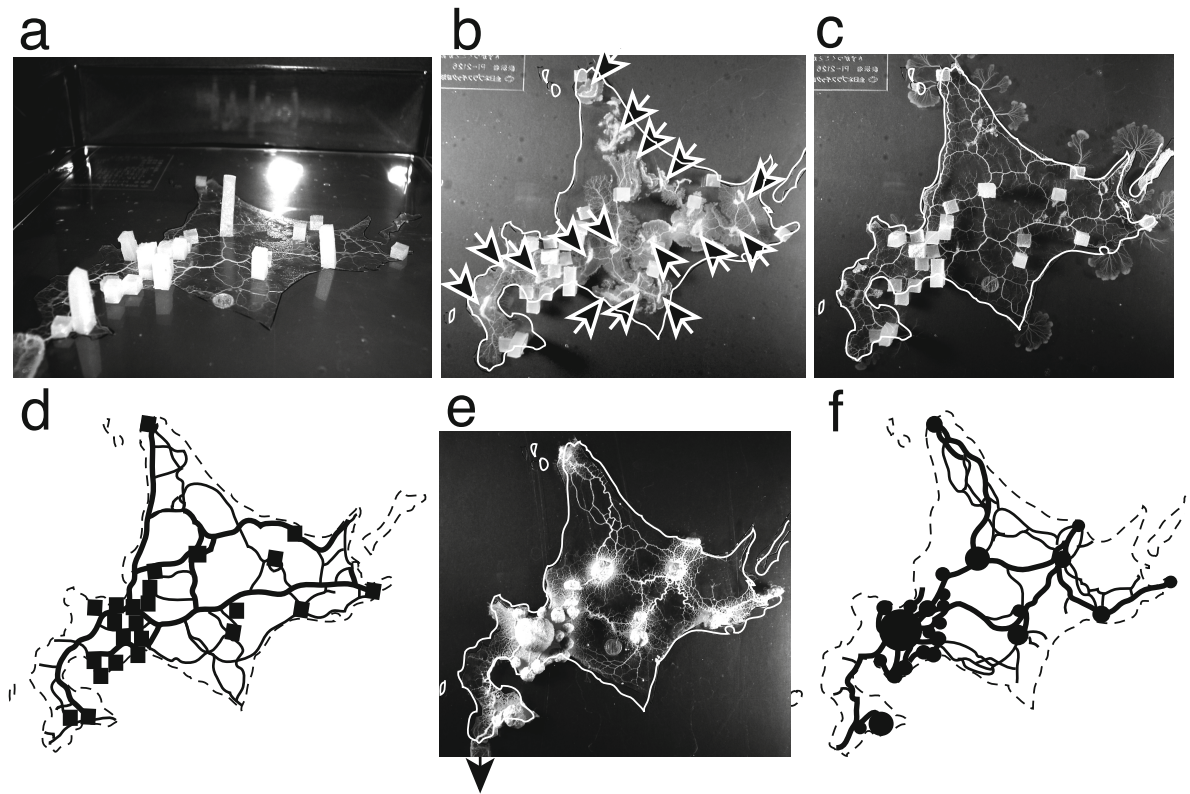

Fig. 6. Time series of the tube-network made by Physarum when presented with different sized FSs. (a) A bird's-eye view of the experimental setup in which each FS was cubic column of agar with constant width, but varying height. In general, the surface area of the block scaled with population size, except for the city of Sapporo which was too high to stand. Thus in this case a smaller block but with more oat powder was used. (b) Intermediate stage of growing network. The arrows indicate the locations of organism initially inoculated. (c) The final network that connected all FSs. (d) The network shape extracted from (c). (e) The final network formed when different diameters of oat dough was used as FSs and (f) the corresponding network shape.

Although the network shape was different in each experimental setup, the functional behaviour measured as the total length of the network and its faulttolerance were similar. The fault-tolerance was defined here as the tolerance of the global connectivity of all FSs to random, accidental disconnection of tube as described previously [8, 11]). These two measures should be traded-off against each other to give a reasonably robust, but not too expensive network. When the size of the FS was varied to reflect population numbers in each location, the skeleton structure remained broadly similar, and still provided a good trade-off between total length and fault-tolerance (Fig. 8).

Figure 7 shows the effects of including mountainous regions on network formation across Hokkaido. Different sized columnar FSs were used as described in Fig. 6, and the mountains were represented by larger blocks positioned to match the four mountain ranges in Hokkaido to prevent the organism exploring these regions (see Fig. 7a). The network structure extracted from Fig. 7b is shown in Fig. 7c. In another experiment, the impact of the mountainous regions was 
a

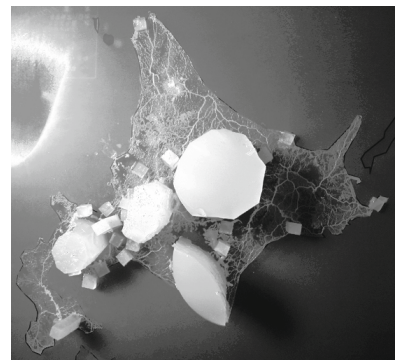

d

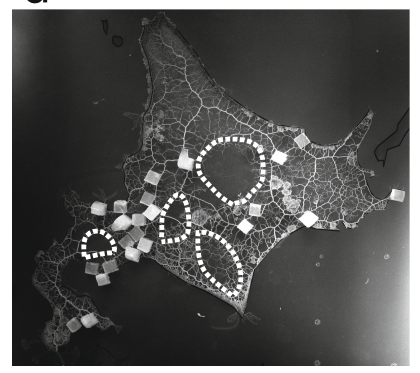

b

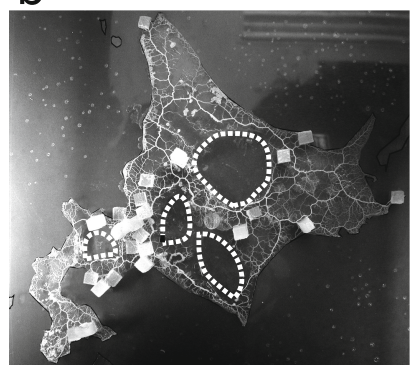

e

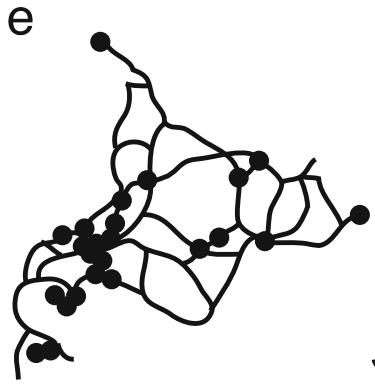

C

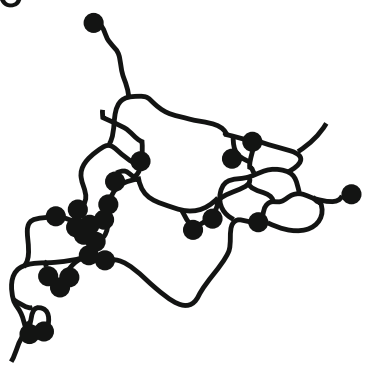

\section{f}

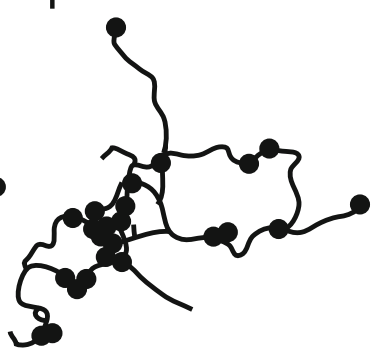

Fig. 7. Effect of including geographical mountainous features on the network formed by Physarum. (a) Top view of the experimental setup. Different sized columnar FSs were used, as described in Fig. 6, but with the inclusion of additional non-nutrient blocks of agar corresponding to the four mountain ranges in Hokkaido. (b) The network shape formed around the obstacles, where the thick white dashed lines indicate the locations of the obstacles that were removed after the experiment. (c) Network shape obtained from (b). (d) Network development under patterned illumination (45000 lux) that mapped onto the four mountain ranges (indicated by the thick white dashed lines). The light illumination discouraged growth through the mountainous areas. (e) Extracted network formed in (d). (f) Network layout of the actual railway lines in Hokkaido region in 2010.

simulated using light illumination which Physarum tends to avoid. A typical network shape is shown in Fig.7d-e. Figure $7 \mathrm{f}$ shows the actual railway network in Hokkaido region in 2010, for comparison with the Physarum networks.

\section{Path-Finding and Multi-functionally Networking Derived by Morphogenetic Dynamics of Vein}

We proposed a simple mathematical model for network formation in the Hokkaido arena. The basic framework of mathematical modeling was extended from the previous model[11] to include the impact of geographical variations of altitude. In brief, the Physarum network is represented as a network of water pipes. Fig. 9a show a schematic illustration of model setup with a series of rigid cylindrical 
pipes connected at junctions, $i$ and $j$. The pipe is characterized by three physical quantities: length $L_{i j}$, radius $r_{i j}$, and flow rate $Q_{i j} . Q_{i j}$ is given by Poisueille flow approximation $Q_{i j}=\frac{\pi r_{i j}^{4}}{8 \eta} \frac{\left(P_{i}-P_{j}\right)}{L_{i j}}$, where $\eta$ is viscosity of fluid, and $P_{i}$ is pressure at the joint $i$.

At each iteration of the simulation, two joints are selected that correspond to FSs. A constant current $Q_{0}$ flows into one of the two and flows out of the other. We can calculate the flow, $Q_{i j}$, through every pipe, as $Q_{0}$ and all $L_{i j}$ and $r_{i j}$ are given, assuming flow follows Kirchoff's law of fluid flow, namely that the sum of currents flowing into each joint is equal to the sum of currents flowing out of that joint.

Next we included the effect of changing vein diameter to simulate the strengthening or disappearance of tubes. The mechanistic basis controlling the dynamics of vein morphogenesis is not well defined, but recent experiments show a strong correlation between the rate of protoplasmic shuttle streaming through the vein itself and its subsequent diameter [15, 16]. Thus veins become thicker when the protoplasmic flow is large enough, but otherwise tend to thin. In essence, each vein adapts locally to the flow through the vein itself that can be described using equations for vein growth [6].

We prefer to use tube conductivity $D_{i j}=\frac{\pi r_{i j}^{4}}{8 \eta}$ rather than just tube radius $r_{i j}$. Dynamic changes in conductivity follow the balance of two antagonistic processes,

$$
\frac{d D_{i j}}{d t}=f\left(\left|Q_{i j}\right|\right)-\alpha D_{i j}
$$

where $f\left(\left|Q_{i j}\right|\right)$ is the thickening process that depends on the protoplasmic flow and $-\alpha D_{i j}$ is the thinning process with first order reaction kinetics. The function $f\left(\left|Q_{i j}\right|\right)$ is a monotonically increasing function of the absolute value of $Q_{i j}$. In this case, we assume $f\left(\left|Q_{i j}\right|\right)=\tanh \left(\left|Q_{i j}\right|-1\right)-\tanh (-1)$. The thinning process comes from the experimental evidence that any local part of organism tends to reduce the intervening network as it accumulates on the FSs.

We now consider the coefficient of the thinning process, $\alpha$, which can be tuned to reflect geographical features, such as the mountain ranges, lakes and the sea. $\alpha$ is proportional to altitude and scales from $[1 \leq \alpha \leq 3]$ over the range 0 to $2000 \mathrm{~m} . \alpha$ is also set to maximum over seas, lakes and rivers.

We would like to stress the point that the tube dynamics are controlled locally, with each vein changing according to its own flow and conductivity alone. Nevertheless, the constant $Q_{0}$ controls the total flow through the network and provides some indirect coupling across the system. The magnitude of $Q_{0}$ plays an important role in the various interactions between the elementary components of these types of models (veins in this case), and is particularly influential on the number of tubes that persist in the simulation. Thus the final network architecture produced by the model is highly dependent on $Q_{0}$ and the form of $f$, as discussed previously [6, 11, 17]. 


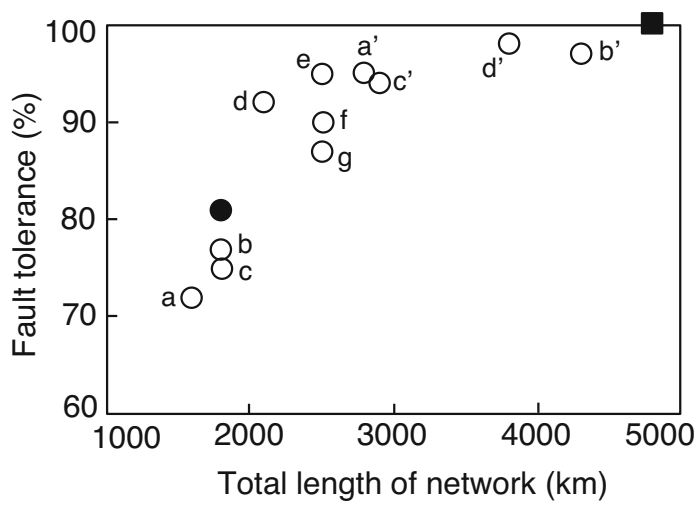

Fig. 8. Evaluation of network performance. Open circles represent experimental data points, with letters representing individual experiments. (a, b) are data from experiments without mountainous regions and equal-sized FSs as shown in Fig. 5; (c, d) include scaling for population size according to Fig. 6a; (e, f) include physical mountainous regions shown in Fig. 7a, whilst (g) includes illumination as shown in Fig. 7d. The 'prime' symbol indicates a data for network that includes the thinner connections, while letters with no prime indicate the skeleton structure of the network with thicker connections. The closed circle and square are results for the real rail and road network, respectively, in Hokkaido region.

\section{Model Simulation}

Figure 9b-e shows the results of the simulation. The network connects all of the FSs, however, depending on the parameter $Q_{0}$, the final shape of network is different, most notably, the total length of network is longer with more parallel paths as $Q_{0}$ increases. Nevertheless, the simulated network obtained by the model overlaps the real rail transportation network shown in Fig 7e-g, for relatively low values of $Q_{0}$. For comparison with the road network, which has a wide range of different categories of road from major highways to minor side roads, roads were assigned a priority based on their level in hierarchy, and added until total length of lines reached the total length in the simulation. We assume that the highest priority were the highways and descending order of number of national roads (Kokudo) next. The network shape was similar between the model and the real road network with increasing $Q_{0}$.

The network tends to avoid the high land, but manages to find routes through the mountain passes acting like a saddle point (indicated by the arrow numbered 1, 2 and 3). Such passes are relatively rare, nevertheless several cities in the south eastern part are connected very effectively with cities in north eastern part of Hokkaido through saddle number 1 , and western central part through saddles 2 and 3 . These correspond to the real saddle points on the railway and main roads of Bihoro-toge, Karikachi-toge and Nissho-toge, respectively, in Hokkaido. 
a
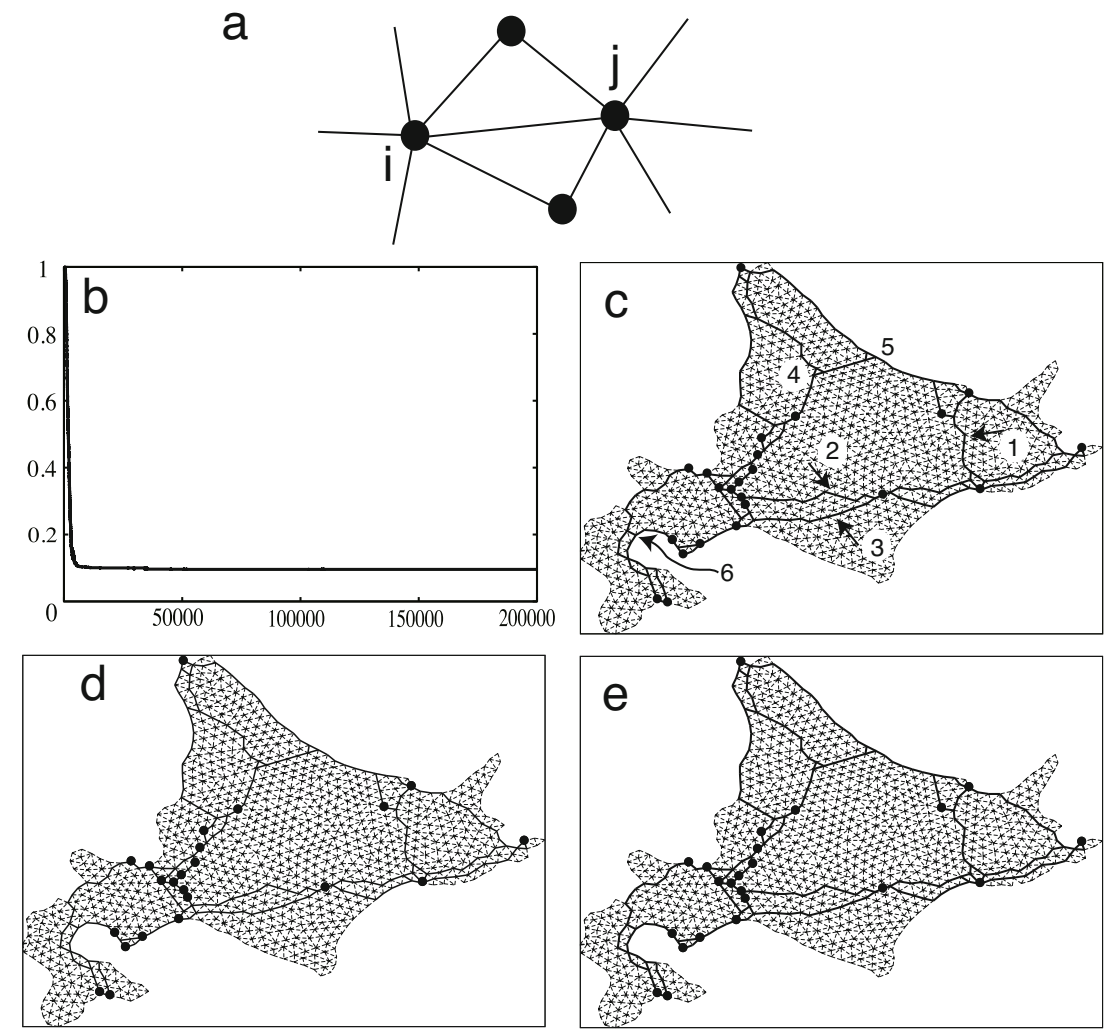

Fig. 9. Model simulation for development of the transportation network between major cities in the Hokkaido region. (a) Schematic representation of the model representing the Physarum plasmodium as network of hard cylindrical water pipes, $i j$, that connect two joints $i$ and $j$. (b) Time course of the total length of network, that initially covered the whole of Hokkaido as a fine mesh, normalized by the length of initial conditions of network. The total length decreased as the edges disappeared (became thinner than a threshold values of $10^{-5}$ ). During the initial decrease there was some fluctuations in the total length, but it was essentially monotonic. (c) A typical example of the network shape when the total length of the simulated network had stabilized at $t=200000$ iterations. The numbered arrows 1-3 indicate the correspondence between the simulated network and actual saddle points through the mountain ranges in Hokkaido at Bihorotoge, Karikachi-toge and Nissho-toge, respectively. The numbered arrows 4-6 indicate the correspondence with actual major triple junctions and the position of accompanying cities of Nayoro, Monbetsu and Oshamanbe, respectively. (d,e) show two solutions run with the same parameters as (c) but with different stochastic choice of sink locations to illustrate that the result is insensitive to this aspect of the simulation. The final network shape is similar in all three cases, and the total length of network is also similar. $Q_{0}=6.0, D_{i j}(t=0)=1.0, \alpha$ is proportional to the altitude of land and $\alpha=1$ $(0 \mathrm{~m})$ and $\alpha=3(2000 \mathrm{~m})$, time step $=0.01$, using the Runge-Kutta method to solve the differential equations. The current source, $Q_{0}$, is fixed at Hakodate city (indicated by the black arrow), and the current sink is chosen stochastically at every iteration, with the choice probability proportional to the population of the city. 
In the model simulation shown in Fig. 9c, there are also major triple junctions in the network that form independently of the major cities set up as FSs. These junctions are indicated by the number 4,5 and 6 . Interestingly the actual cities of Nayoro, Monbetsu and Oshamanbe exist at these three locations. From a population point of view, Nayoro and Monbetsu are the next major cities in the ranking following the 23 cities used in the experimental systems and model simulation. It is remarkable that the model simulation mimics the actual transportation network, and predicts the geographical locations of other cities not explicitly specified in the initial conditions.

\section{Discussion}

It was confirmed that Physarum was able to find the shortest connection through topologically similar mazes set out with different physical layouts (Fig. 3). However, deeper exploration of the maze-solving ability of Physarum led to some slightly surprising results. When presented with a series of parallel paths that were similar in length, the notionally equivalent shortest paths were not necessarily selected with equal probability. Thus, the parallel paths $a_{1}$ and $a_{2}$ in Fig 2a were selected with very different probabilities, biased strongly towards $a_{2}$. In contrast, the parallel paths, $b_{1}, b_{2}$ and $b_{3}$ in Fig $2 \mathrm{~b}$ were chosen with equal probability. Taken together, these results suggest that additional factors may influence path selection for the real organism. These factors are also not present in the mathematical model, which gives an equal weighting to paths of the same length. It is possible that path $a_{2}$ is preferred because the actual length traversed by the organism is in fact marginally less than the notional length of the corridor, as the tube can follow a slight diagonal route between the corners and keep to the inner curve around the turns. Thus, path $a_{2}$ has 7 turns, whilst there are only two in the paths $a_{1}$ and $a_{3}$. If this is the case, then Physarum is able to discriminate a difference in path length of only a few percent of the total. This hypothesis is supported by the preliminary evidence that no preference was observed between three paths in the maze given in Fig. 2d where the number of turning points is the same. It is also possible that topographic features, such as the sharper edges of the turns, provide some additional thigmotropic stimulus, leading to preferential selection of such routes. More comparison between the experiment and the mathematical model is needed to discriminate between these possibilities and determine whether Physarum is indeed always able to find the absolute shortest path.

When allowed to form connections between multiple FSs in an arena without constrained paths set up to mimic Hokkaido, the transport network made by Physarum showed similar properties to the real infrastructure networks based on total length and fault tolerance, irrespective of (1) the initial locations of the organism; (2) the relative sizes of the FSs; and (3) the different configurations of the FSs (cubic column with different height but same concentration of nutrient, and dough of oat with different diameter). We infer that the core skeleton structure formed by Physarum is robust against variations of experimental methods and conditions, provided the physical layout of the FSs is kept constant. 
When looking at the core skeleton structure, we tended to ignore the thinner connections, not least because these tended to disappear over extended time periods. However, these connections may be important for the real Physarum as they occupy territory and allow the organism to respond to addition or removal of resources. Thus with the stochastic arrival of a new FS, the thin connections may thicken and become part of core skeleton structure. We expect that the thinner connections can work as potential preparation for unknown variations of external conditions in the future.

The total amount of organism initially present is a major factor that determines the extent of the final network. The volume was not controlled precisely in these experiments, but we estimate the variation was within $\pm 50 \%$. Under these conditions, The core skeleton structure was similar, with most of the variation in the thinner connections. To experimentally delete the thinner connections, we sometimes put a large FS out side the experimental arena. For instance, in Fig. 6e, the black arrow indicates the location of the large FS. After addition of such a large FS, the thinner tubes disappeared, leaving only the thick tubes. In general, thinner tubes reacted more rapidly to perturbations in the environment, whilst the core skeleton tended to be well conserved and persistent.

The mathematical modeling for the transport network in Hokkaido reproduces the real rail (core) and road infrastructure networks remarkably well. It is impressive that the model reproduces the actual saddle points of Bihorotoge, Karikachi-toge and Nissho-toge in Hokkaido, which are the most important routes in the actual network connecting the major cities all over Hokkaido. Another interesting feature is that the model can predict the key triple junctions in the network and the accompanying cities of Nayoro, Monbetsu and Oshamanbe. Overall, these degree of correspondence described above implies that similar underlying mechanisms may be common in the social dynamics of humans and amoebae, based on relatively simple principles of the dynamic interplay between the structure of the networks formed and the transport dynamics that unfold upon them. The elegant solutions found by Physarum underscore its utility to study how natural self-organized systems can create functionality.

Lastly, we discuss difference of network shape among the experiments, the model simulations and the real configuration of roads/rails. In the experiments, a network shape was totally different case by case if we exactly quantitate the position of each line of trail according to the fixed coordinate axis of Hokkaido space. What this paper showed was that the network shapes were similar even in such variety when we viewed from the evaluation measures of balance between the total length and fault tolerance.

Another point of similarity was that case-by-case fluctuations of passage line coming across the mountain range was relatively smaller in repeats of the experiment, the simulation and the real situations. This means that the constraints of landscape like mountains plays a key role in all of three systems.

We noticed differences of network shape one-by-one. For instance, in the real situations, traffic network covers well around the southern part of Hokkaido including the cities of Hakodate, Esashi, Kaminokuni, Matsumae, but it did not 
appear both in the model and the experiments. This may be because we ignored the historical process of development in Hokkaido. In fact, these cities were built at the first stage and frontier wave of development propagated from there in some sense.

We pointed out the other factor to be considered although we neglected in this paper. Hokkaido is not isolated in the ocean but have been connected to neighbor islands by huge traffic of matters and people through ship transport. North-east end (Nemuro, Kushiro) and North end (Wakkanai) are such examples and real traffic network is denser than that in the Physarum experiment and the model simulation.

In the future, we need a new measure that can characterize the functionality and the physical nature of transport network. Similarity and differences may be much more clarified in the real network and Physarum network. Many measures are already proposed in graph theory and so-called network science and, especially, a measure from weighted graph theory, which is not yet developed enough, is to be involved.

Acknowledgements. This research was supported by JSPS KAKENHI 20300105 and by Strategic Japanese-Swedish Research Cooperative Program, Japan Science and Technology Agency (JST).

Open Access. This chapter is distributed under the terms of the Creative Commons Attribution Noncommercial License, which permits any noncommercial use, distribution, and reproduction in any medium, provided the original author(s) and source are credited.

\section{References}

1. Nakagaki, T.: Ph. D. thesis in Nagoya University, Japan (1997), http://eprints.lib.hokudai.ac.jp/dspace/handle/ 2115/34739?locale=en\&lang=en

2. Nakagaki, T., Yamada, H., Tóth, Á.: Maze-solving by an amoeboid organism. Nature 407, 470 (2000)

3. Nakagaki, T., Yamada, H., Tóth, Á.: Path finding by tube morphogenesis in an amoeboid organism. Biophys. Chem. 92, 47-52 (2001)

4. Nakagaki, T.: Smart behavior of true slime mold in labyrinth. Res. Microbiol. 152, 767-770 (2001)

5. Tero, A., Kobayashi, R., Nakagaki, T.: Physarum solver -A biologically inspired method for road-network navigation-. Physica A363, 115 (2006)

6. Tero, A., Kobayashi, R., Nakagaki, T.: Mathematical model for adaptive transport network in path finding by true slime mold. J. Theor. Biol. 244, 553-564 (2007)

7. Nakagaki, T., Yamada, H., Hara, M.: Smart network solutions in an amoeboid organism. Biophys. Chem. 107, 1-5 (2004)

8. Nakagaki, T., Kobayashi, R., Ueda, T., Nishiura, Y.: Obtaining multiple separate food sources: behavioral intelligence in the Physarum plasmodium. Proc. R. Soc. Lond. B 271, 2305-2310 (2004) 
9. Tero, A., Yumiki, K., Kobayashi, R., Saigusa, T., Nakagaki, T.: Flow-network adaptation in Physarum amoebae. Theory in Biosciences 127, 89-94 (2008)

10. Tero, A., Nakagaki, T., Toyabe, K., Yumiki, K., Kobayashi, R.: A method inspired by Physarum for solving the Steiner problem. International Journal of Unconventional Computing 6, 109-123 (2010)

11. Tero, A., Takagi, T., Saigusa, T., Ito, K., Bebber, D.P., Fricker, M.D., Yumiki, Y., Kobayashi, R., Nakagaki, T.: Rules for biologically-inspired adaptive network design. Science 327, 439-442 (2010)

12. Nakagaki, T., Saigusa, T., Tero, A., Kobayashi, R.: Effects of food amount on path selection in transport network of an amoeboid organism. Topological Aspects of Critical Systems and Networks, 94-100 (2007)

13. Watanabe, S., Tero, A., Takamatsu, A., Nakagaki, T.: Traffic optimization in railroad networks using an algorithm mimicking an amoeba-like organism. Physarum Plasmodium, Biosystems 105, 225-232 (2011)

14. Nakagaki, T., Iima, M., Ueda, T., Nishiura, Y., Saigusa, T., Tero, A., Kobayashi, R., Showalter, K.: Minimum-risk path finding by an adaptive amoebal network. Phys. Rev. Lett. 99, 068104 (2007)

15. Nakagaki, T., Yamada, H., Ueda, T.: Interaction between cell shape and contraction pattern. Biophys. Chem. 84, 195-204 (2000)

16. Nakagaki, T., Guy, R.: Intelligent behaviors of amoeboid movement based on complex dynamics of soft matter. Soft Matter 4, 1-12 (2008)

17. Nakagaki, T., Tero, A., Kobayashi, R., Onishi, I., Miyaji, T.: Computational ability of cells based on cell dynamics and adaptability. New Generation Computing 27, 57-81 (2008) 